3. Pallares CJ, Martínez E. Implementation of a regulated antibiotic use program in two medical-surgical intensive care units in a thirdlevel teaching hospital in Colombia. Infectio 2012;16:192-198.

4. Hernández-Gómez C, Pallares C, Escandón-Vargas K, et al. Economic impact of an antimicrobial stewardship program implementation in three high-complexity hospitals in Colombia. Open Forum Infect Dis 2016;3:S273.

5. Cabana MD, Rand CS, Powe NR, et al. Why don't physicians follow clinical practice guidelines? A framework for improvement. JAMA 1999;282:1458-1465.

6. Goldstein EJC, Goff DA, Reeve W, et al. Approaches to modifying the behavior of clinicians who are noncompliant with antimicrobial stewardship program guidelines. Clin Infect Dis 2016;63:532-538.

\section{Deadly Sins of Antibiotic Abuse in China}

To the Editor-China ranks first among the world's most frequent users of antibiotics. ${ }^{1}$ In the hospital setting, $\sim 70 \%$ of inpatients and $\sim 20 \%$ of outpatients in China are prescribed antibiotics by doctors, which are both twice WHO's expectation for these rates. ${ }^{2}$ In many developed countries and in the United States, these rates are $\sim 30 \%$ of inpatients and $\sim 15 \%$ of outpatients. The overuse of antibiotics not only may adversely affect their therapeutic efficacy but also may substantially increase the cost of health care and lead to lethal toxic or allergic reactions. Approximately 14,738,000 incidents of moderate-to-severe adverse effects related to antibiotic abuse are reported annually in China, and between 2001 and 2005, 150,000 patients died annually of antibiotic abuse or overuse. ${ }^{3}$ Moreover, off-label and blind use of antibiotics without pathogen culture and sensitivity test can lead to increased antimicrobial resistance. ${ }^{4}$ Furthermore, the case-fatality rate of drug-resistant infections reached $11.7 \%$ in hospitalized patients in China in $2011 .^{5}$ Based on the data provided in the Mohnarin report, ${ }^{6}$ between 2007 and 2008 the detection rates of methicillin-resistant Staphylococcus aureus (MRSA) and methicillin-resistant Staphylococcus epidermidis (MRSE) were $73.6 \%$ and $79.5 \%$, respectively, in 20 hospitals in China. These rates are by far the highest in history.

The irrational clinical practice of antibiotic use in China is mainly characterized by high frequency and intensity, long duration, high dose, abuse without definite indications, and the combination of multiple antibiotics without the support of evidence. The "7 deadly sins" behind antibiotic abuse in China include (1) blind dependence on antibiotics on the part of the patient, (2) lack of awareness of proper use of antibiotics on the part of the doctor, (3) compulsory acquisition of interest on the part of the hospital, (4) enormous loopholes in the drug sale and purchase market, (5) lack of strict management systems, (6) ineffective administrative supervision, ${ }^{7-9}$ and (7) longstanding hospital systems that compensate doctors according to their prescription practices. In addition, antibiotic abuse in animal husbandry is almost an open secret in China. Some scholars worry that the Chinese people will regret these lax antimicrobial policies and that China may even enter a "post-antibiotic era" in which antibiotics are not longer effective, which would undoubtedly constitute a humanitarian disaster.

Antibiotic abuse and other pressing public health challenges have attracted the attention of policy makers in the Chinese government. In May 8, 2012, the Chinese government officially issued an "unprecedentedly strict" (the networkbased language prevailing in China) document concerning the management of clinical application of antibiotic agents. Mr. Xiaowei Ma, Deputy Minister of the Ministry of Health of China, said that the Ministry of Health would take further measures to curb antibiotic abuse (1) by including further intensifying the management of clinical application of antibiotic agents by setting up relevant policies, (2) by strictly implementing regulations about staged management of antibiotic agents and the prescription review system, (3) by progressively enhancing the construction of networks on clinical application of antibiotics and supervision of dynamic surveillance as well as early warning on antibiotic resistance, and (4) by immediately taking specific actions to rectify improper clinical application of antibiotic agents. ${ }^{10}$

Beyond China, antibiotic abuse has become a serious social issue threatening public health all over the world. We need to raise public awareness about the harmful nature of antibiotic abuse. Media should emphasize the importance of the rational use of antibiotics, and measures should be taken to improve public knowledge of both risks and benefits of antimicrobial therapy. In addition, education of doctors should be improved to ensure that they comply with such guidelines as well as the widely accepted recommendations for proper clinical diagnosis and treatment. Returning public hospitals to the track of public service, mobilizing the enthusiasm of medical workers, and preventing them from abusing antibiotics for personal gains are key to achieving the rational use of antibiotics.

\section{ACKNOWLEDGMENTS}

Financial support: No financial support was provided relevant to this article.

Potential conflicts of interest: All authors report no conflicts of interest relevant to this article.

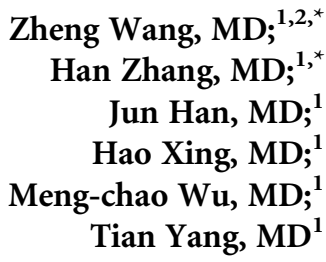

Affiliations: 1. Eastern Hepatobiliary Surgery Hospital, Second Military Medical University, Shanghai, China; 2. Department of Clinical Medicine, Second Military Medical University, Shanghai, China. 
Address correspondence to Dr Tian Yang, Eastern Hepatobiliary Surgery Hospital, Second Military Medical University, Shanghai 200438, China (yangtian6666@hotmail.com).

* These two authors contributed equally to this work. Infect Control Hosp Epidemiol 2017;38:758-759

(C) 2017 by The Society for Healthcare Epidemiology of America. All rights reserved. 0899-823X/2017/3806-0026. DOI: 10.1017/ice.2017.60

\section{REFERENCES}

1. Xiao YH. Bacterial resistance: challenge and strategies. China Lic Pharm 2011;8:3-8 (in Chinese).

2. Li YB, Xu J, Wang F, et al. Overprescribing in China, driven by financial incentives, results in very high use of antibiotics, injections, and corticosteroids. Health Affairs 2012;31:1075-1082.

3. Xiao Y, Hou F, Wang J, et al. An investigation into socioeconomic impact of adverse drug reactions of antibacterial agent irrational use. Chin Health Econ 2010;29:94-96 (in Chinese).

4. Zhang R, Eggleston K, Rotimi V, Zeckhauser RJ. Antibiotic resistance as a global threat: evidence from China, Kuwait and the United States. Global Health 2006;2:6.

5. Yang F, Liu SM. Attention to antibiotic resistance: the nightmare from medicine industry to food industry. Legal Syst Soc 2011;279: 56-58 (in Chinese).

6. Li Y, Liu J, Xue F, et al. Antimicrobial susceptibility surveillance in China: a Mohnarin program 2007-2008 report. Chin J Clin Pharmacol 2011;27:323-334 (in Chinese).

7. Currie J, Lin W, Zhang W. Patient knowledge and antibiotic abuse: evidence from an audit study in China. J Health Econ 2011;30:933-949.

8. Chen M. Analysis on the condition of, reasons for and control on antibiotic abuse. Jiangsu Health Care Manage 2005;16:49-50 (in Chinese).

9. Ma Y. Rational use of antibiotics to inhibit the extension of bacterial resistance. China Pharm 2011;26:2401-2403 (in Chinese).

10. Wang CJ. Combat antibiotic abuse with multiple measures. Chin J Health 2011;5:40-41 (in Chinese).

\section{Gunshot Injury Paraplegics-A Population Dying a Slow, Irreversible, and Expensive Death-A Viewpoint on Preventing Pressure Ulcers}

To the Editor-Over the last 20 years, healthcare expenditure in the United States has been a subject of immense national interest. Given the increase in healthcare spending $(>15 \%$ of the gross domestic product $\left.{ }^{1}\right)$, healthcare agencies are giving more attention to disease prevention in a variety of healthcare areas including infection prevention, antibiotic stewardship, and prevention of pressure ulcers.

While clinicians are very familiar with pressure ulcers in elderly bedridden patients, pressure ulcers are also a serious complication among spinal cord injury (SCI) paraplegics. The annual incidence of pressure ulcers in SCI paraplegics has been reported to be $23 \% .^{2}$ In the United States, most SCIs are an unfortunate result of gunshot injuries; this is especially true in metropolitan Detroit. Annually, a major tertiary-care hospital in Detroit treats 40-60 gunshot SCI paraplegic patients (GIPs) with pressure ulcers. ${ }^{3}$ A recent study conducted at the Detroit Medical Center reported that 201 gunshot-SCI patients with pressure ulcers accounted for 395 admissions (including readmissions) between 2004 and 2008. During this study period, the cumulative median length of hospital stay per patient was 12 days (interquartile range, [IQR], 6-24 days), resulting in a mean adjusted cost of US\$19,969 ( $\pm \$ 6,639)$ per patient. ${ }^{3}$

The number of GIPs is growing exponentially, ${ }^{4}$ and GIPs with pressure ulcers are frequently admitted to the hospital, thus contributing significantly to hospital costs. The economic burden of treating pressure ulcers in this population is enormous but underappreciated. The annual cost of treating pressure ulcers in the United States in paraplegics with or without SCIs has reached a stunningly high $\$ 11$ billion. ${ }^{5}$ The estimated cost associated with healing a single pressure ulcer can reach $\$ 40,000 .^{5}$ Are government officials knowledgeable enough and aware of this enormous economic burden? Are healthcare professionals equipped to deal with this unfortunate patient population?

Let us consider the following scenarios of 2 gunshot SCI paraplegic patients at a tertiary-care hospital in Detroit: These 2 gunshot SCI paraplegic patients were admitted with pressure ulcers; both were male African Americans in their late 30s and belonged to low-education and low-income demographic groups. Despite their similarities in age and circumstance, their hospital costs were dramatically different. Costs for hospitalization for the first patient were moderate at $\$ 46,300$, whereas the hospital costs for the second patient reached $\$ 262,168$. The higher costs for the second patient resulted from an infected pressure ulcer: this patient was readmitted 3 times, and his treatment included several diagnostic procedures (including MRIs) and surgical interventions (ie, incision and drainage).

Development of a pressure ulcer in a paraplegic patient can be devastating socially, emotionally, and financially. An American soldier with a SCI is cared for in a very supportive environment. On the other hand, an African-American GIP residing in Detroit with low education, low income, and a poor support system has very limited resources to support his physical and mental health. Such destitute patients in the inner city are living slow but irreversible and expensive death sentences.

Through value-based purchasing, the Centers for Medicare and Medicaid Services (CMS) has begun to decrease payments to hospitals with excessive 30-day readmissions. The CMS will not provide hospitals added insurance reimbursement when pressure ulcers develop in the hospital. With the advent of these new regulations, what are we doing to prevent pressure ulcers in GIPs? In the last decade, both medical and surgical treatment modalities for pressure ulcers have progressed remarkably. However, there are major deficiencies in preventing pressure ulcers in GIPs.

Healthcare reforms should include more incentives for prevention and early intervention of pressure ulcers among GIPS. 